\title{
More than meets the eye: esophageal invasive mucinous adenocarcinoma masquerading as a submucosal lipoma on endoscopic ultrasound
}

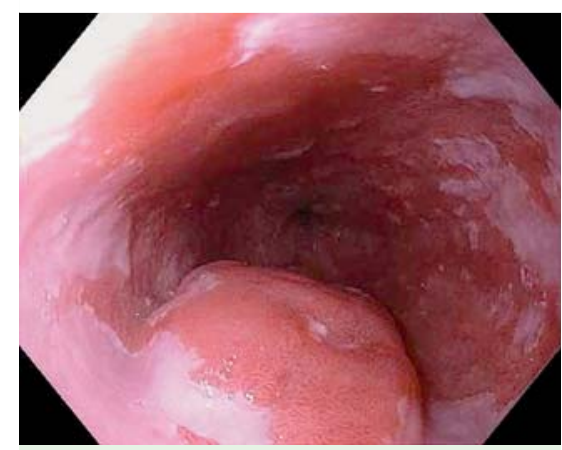

Fig. 1 Subepithelial lesion arising in the distal esophagus underneath a segment of Barrett's mucosa.

Endoscopic ultrasound (EUS) is considered to be the most accurate method of locoregional staging of advanced but potentially curable esophageal lesions [1]. This report describes a case of a mucinous adenocarcinoma arising in the setting of Barrett's esophagus with benign endosonographic features mimicking a lipoma.

A 67-year-old man was referred for evaluation of Barrett's esophagus (Prague Criteria C5M5) with foci of high grade dysplasia overlying a subepithelial lesion ( $\mathbf{F i g} \mathbf{1}$ ). EUS revealed a hyperechoic subepithelial lesion with well-defined borders originating from the submucosa ( $\mathbf{F i g . 2}$ ), endosonographic features that are most consistent with a lipoma. In the presence of this lesion, it was thought that the raised overlying Barrett's dysplastic mucosa would not be adequately treated by radiofrequency ablation. Thus, endoscopic submucosal dissection (ESD) was performed, keeping the submucosal dissection plane just above the proper muscle layer in an effort to remove the submucosal lesion and overlying Barrett's mucosa en bloc (๑ Fig. 3).

In contrast to the initial negative mucosal biopsies and benign endosonographic appearance, histology surprisingly revealed a moderately differentiated mucinous adenocarcinoma without lymphovascular invasion, with focal invasion into the submucosa (stage pT1bNx) and negative lateral and deep resection margins (R0 resection). The submucosal lesion, thought to be a lipoma based on EUS appearance, consisted of a well-circumscribed tumor

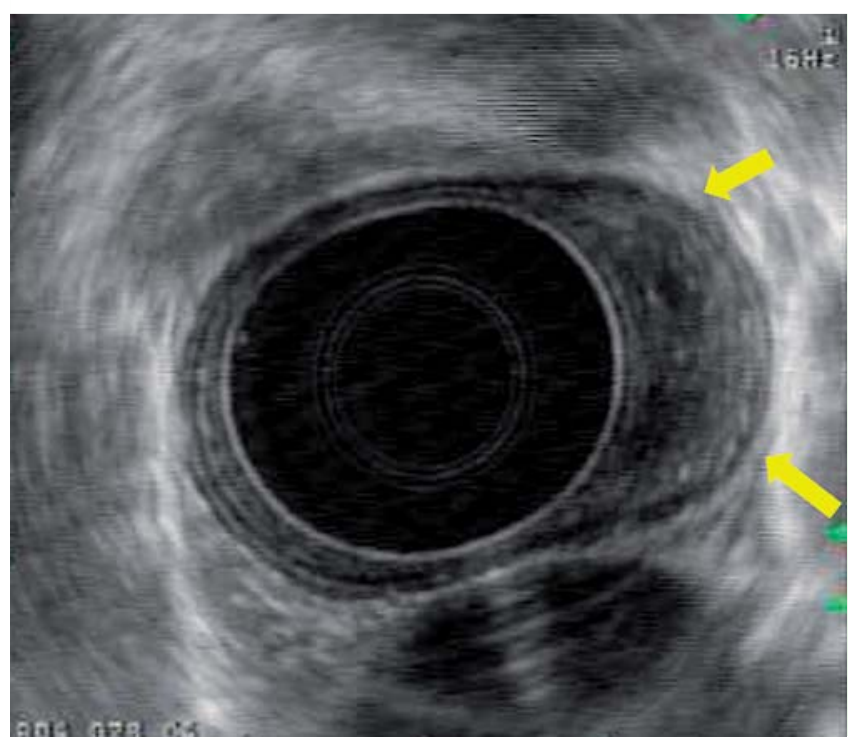

Fig. 2 Endoscopic ultrasound image showing hyperechoic lesion with well-defined borders (arrows) arising from the third layer of the esophagus.

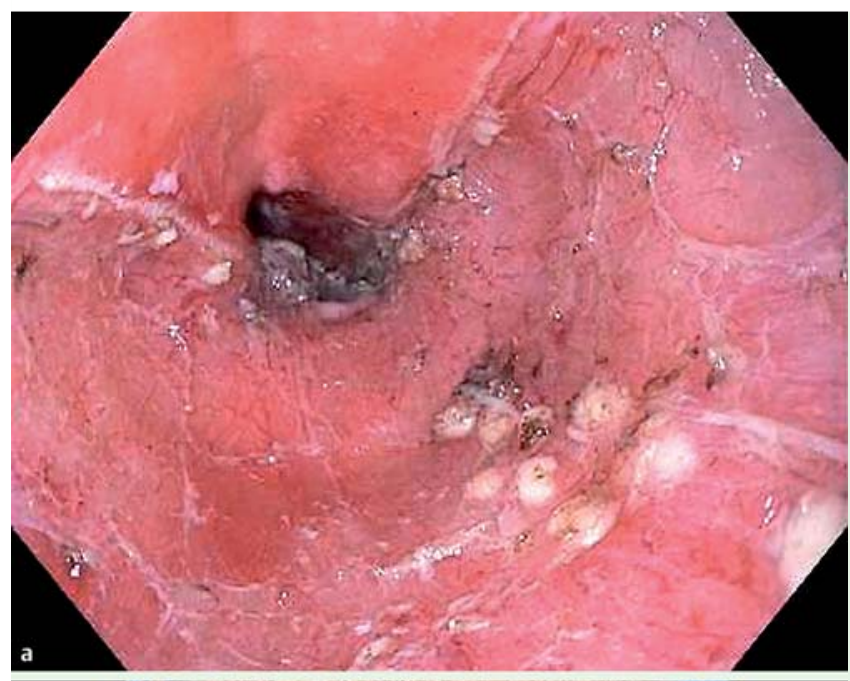

Fig. 3 Endoscopic view. a The mucosal defect following endoscopic submucosal dissection. $\mathbf{b}$ Subepithelial lesion and Barrett's mucosa removed en bloc.

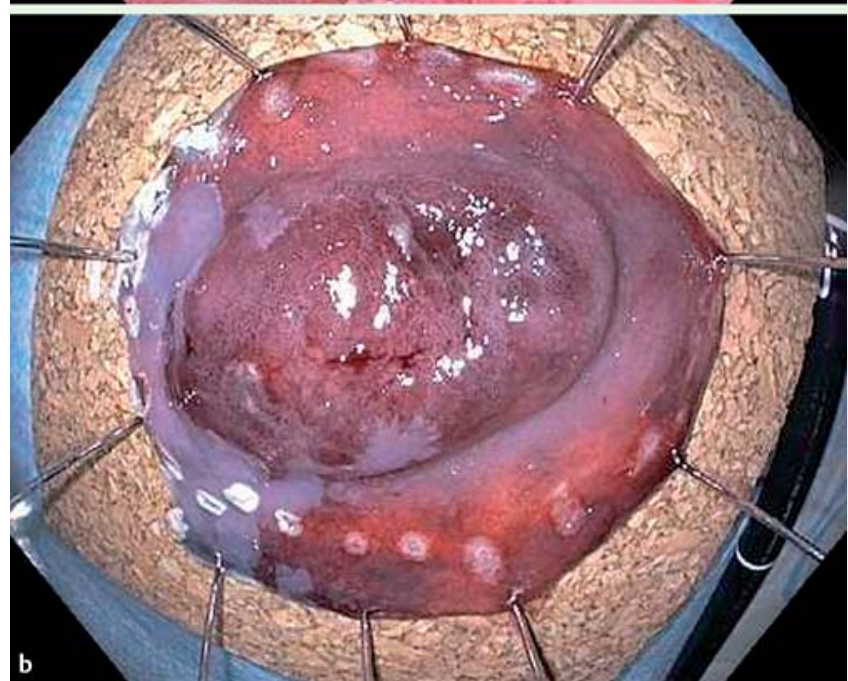




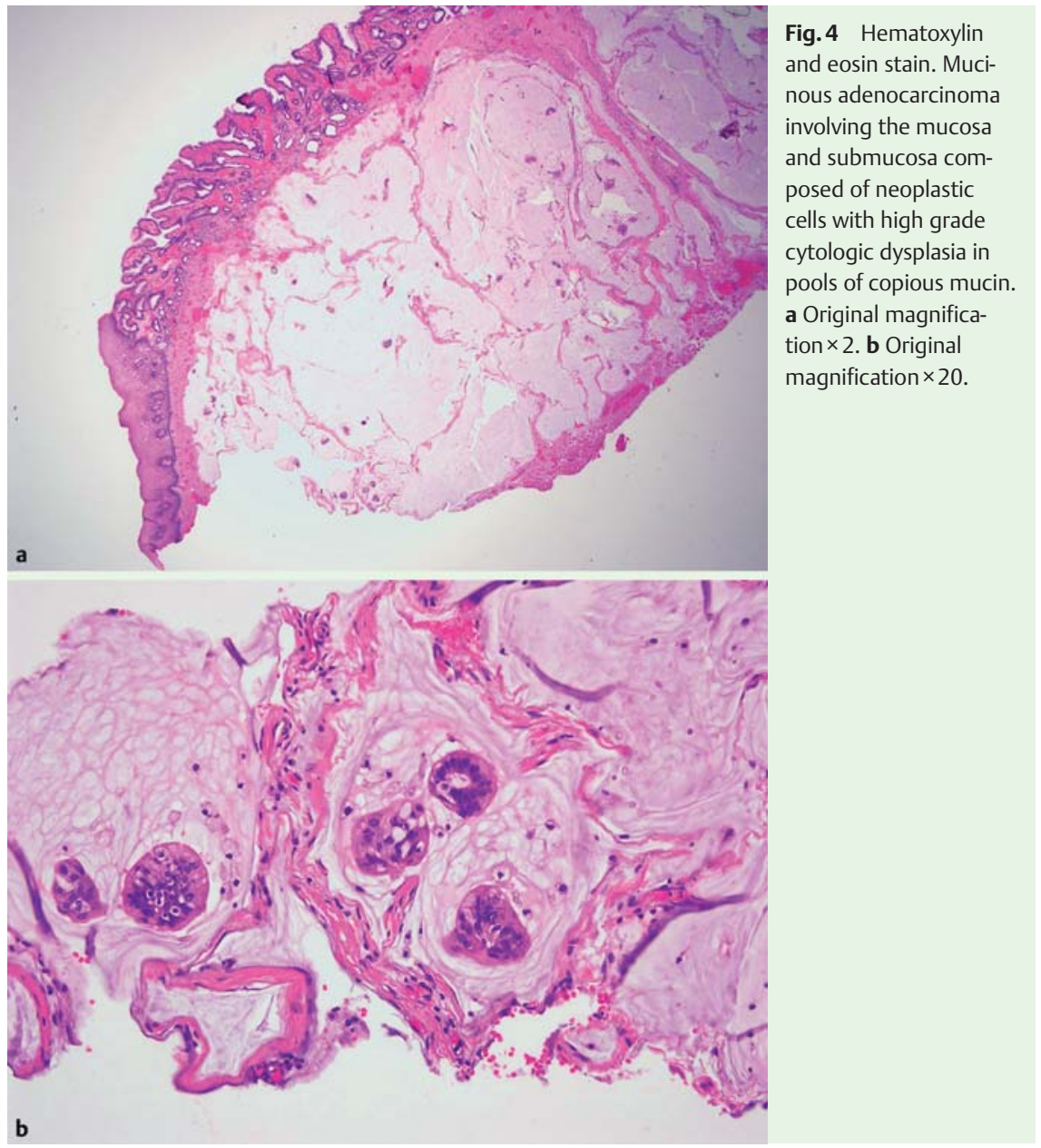

with pools of mucin that contained neoplastic cells with high grade cytologic atypia ( $\bullet$ Fig.4).

EUS is regarded as the best imaging technique for diagnosing and differentiating between submucosal lesions in the gastrointestinal tract [2]. Nonetheless, malignancy can occasionally masquerade as a benign etiology on imaging alone, high-
Endoscopy_UCTN_Code_CCL_1AB_2AC_3AB

Competing interests: None

Dennis Yang1, Lizette Vila Duckworth ${ }^{2}$, Peter V. Draganov ${ }^{1}$

${ }^{1}$ Department of Medicine, Division of Gastroenterology and Hepatology, University of Florida, Gainesville, Florida, United States

${ }^{2}$ Department of Pathology, University of Florida, Gainesville, Florida, United States

\section{References}

1 Thosani N, Singh H, Kapadia A et al. Diagnostic accuracy of EUS in differentiating mucosal versus submucosal invasion of superficial esophageal cancers: a systematic review and meta-analysis. Gastrointest Endosc 2012; 75: $242-253$

2 Rosch T, Kapfter B, Will U et al. Endoscopic ultrasonography. Accuracy of endoscopic ultrasonography in upper gastrointestinal submucosal lesions: a prospective multicenter study. Scand J Gastroenterol 2002; 37: $856-862$

Bibliography

DOI http://dx.doi.org/ 10.1055/s-0034-1377764

Endoscopy 2014; 46: E502-E503

(c) Georg Thieme Verlag KG

Stuttgart · New York

ISSN 0013-726X

lighting the importance of clinical correlation and pursuing additional evaluation when indicated. As demonstrated in this case, ESD can provide accurate clinical diagnosis and histopathologic staging that is remarkably different from the initial diagnosis based on forceps biopsy and EUS imaging.

\section{Corresponding author \\ Peter V. Draganov, MD}

Division of Gastroenterology,

Hepatology and Nutrition

Department of Internal Medicine

University of Florida

1329 SW 16th Street, Room \#5251

Gainesville FL 32608

United States

Fax: +1-352-392-3618

Peter.draganov@medicine.ufl.edu 